\title{
CHAINS AND DISCRETE SETS IN ZERO-DIMENSIONAL COMPACT SPACES
}

\author{
MURRAY BELL AND JOHN GINSBURG ${ }^{1}$
}

\begin{abstract}
Let $X$ be a compact zero-dimensional space and let $B(X)$ denote the Boolean algebra of all clopen subsets of $X$. Let $\kappa$ be an infinite cardinal. It is shown that if $B(X)$ contains a chain of cardinality $\kappa$ then $X \times X$ contains a discrete subset of cardinality $\kappa$. This complements a recent result of $J$. Baumgartner and $P$. Komjath relating antichains in $B(X)$ to the $\pi$-weight of $X$.
\end{abstract}

1. Introduction. Let $X$ be a compact zero-dimensional space, and let $B(X)$ denote the Boolean algebra of clopen subsets of $X$. In this note we are interested in the relations between certain algebraic aspects of the Boolean algebra $B(X)$ and the topological properties of $X$. Specifically, we are concerned with chains and antichains in $B(X)$ and their connection with cardinal invariants of the space $X$.

Our set-theoretic and topological terminology and notation are standard. The cardinality of a set $S$ is denoted by $|S|$. All cardinal numbers considered in this paper are assumed to be infinite. Our basic reference for cardinality properties of topological spaces is [ $\mathbf{J}]$.

For the reader's convenience we now recall several notions which will be useful in the sequel.

Recall that a topological space $D$ is said to be discrete if every point of $D$ is open in $D$. Thus if $D$ is a subspace of a space $X$, then $D$ is discrete if for every point $x$ of $D$ there is an open set $G_{x}$ in $X$ such that $G_{x} \cap D=\{x\}$. If $X$ is a topological space the spread of $X$, denoted by $s(X)$, is defined by

$$
s(X)=\sup \{\kappa: X \text { contains a discrete subset of cardinality } \kappa\} .
$$

If $X$ is a space then the density character of $X$, denoted by $d(X)$, is defined by $d(X)=\min \{\kappa: X$ has a dense subset of cardinality $\kappa\}$. If $d(X)=\omega$ we say that $X$ is separable. The hereditary density character of $X$, denoted by $\bar{d}(X)$, is defined by $\bar{d}(X)=\sup \{d(S): S \subseteq X\}$. If $\bar{d}(X)=\omega$ we say that $X$ is hereditarily separable.

A family $\Pi$ of nonempty open subsets of a space $X$ is called a $\pi$-base for $X$ if every nonempty open subset of $X$ contains a member of $\Pi$. The $\pi$-weight of $X$, denoted by $\pi(X)$, is defined by $\pi(X)=\min \{\kappa: X$ has a $\pi$-base of size $\kappa\}$. The hereditary $\pi$-weight of $X$, denoted by $\bar{\pi}(X)$, is defined to be

$$
\bar{\pi}(X)=\sup \{\pi(S): S \subseteq X\}
$$

Received by the editors October 15, 1980 and, in revised form, January 21, 1981.

1980 Mathematics Subject Classification. Primary 54A25; Secondary 54030.

Key words and phrases. Discrete set, compact zero-dimensional space, chain, antichain, strong antichain.

${ }^{1}$ The second author dedicates his research in this paper to Sharon and Gillian. 
Clearly if $D$ is a discrete space of cardinality $\kappa$ then the density character of $D$ is $\kappa$. Therefore $s(X) \leqslant \bar{d}(X)$. If $\Pi$ is a $\pi$-base for $X$ of cardinality $\kappa$ and if for each $P \in \Pi$ we choose a point $x_{P}$ in $P$, then the set $D=\left\{x_{P}: P \in \Pi\right\}$ is a dense subset of $X$ of cardinality at most $\kappa$. This shows that $d(X)<\pi(X)$ and hence that $\bar{d}(X)<\bar{\pi}(X)$.

Let $X$ be a space and let $(T, \leqslant)$ be a totally ordered set. Let $S=\left\{x_{t}: t \in T\right\}$ be a subset of $X$ indexed by $T$. $S$ is said to be totally separated by $T$ if $\left\{x_{s}: s<t\right\}$ is open in $S$ for every $t$ in $T$. We say that $S$ is a totally separated subspace of $X$.

If $B$ is a Boolean algebra then elements $x$ and $y$ of $B$ are said to be comparable if either $x<y$ or $y \leqslant x$; otherwise $x$ and $y$ are said to be incomparable. A subset $S$ of $B$ is called a chain if every two elements of $S$ are comparable. A subset $S$ of $B$ is called an antichain if no two elements of $S$ are comparable. Finally, a subset $S$ of $B$ is called a strong antichain if no element $x$ of $S$ is contained in the union of any finite subset of $S-\{x\}$; that is, $x \nless \bigvee F$ for any finite subset $F$ of $S-\{x\}$. Every strong antichain is an antichain but not conversely. The height of $B$ and the width of $B$ are defined respectively by $h(B)=\sup \{\kappa: B$ contains a chain of cardinality $\kappa\}$ and $w(B)=\sup \{\kappa: B$ contains an antichain of cardinality $\kappa\}$.

2. Chains in $B(X)$ and discrete subsets of $X \times X$. Throughout this section we assume that $X$ is a zero-dimensional compact space.

In [BK] it is shown that if all antichains of $B(X)$ have cardinality at most $\kappa$ then $X$ has a $\pi$-base of cardinality at most $\kappa$. That is, $\pi(X)<w(B(X))$. In [IN] it is pointed out that this result actually holds for hereditary $\pi$-weight: $\bar{\pi}(X)<$ $w(B(X))$. In particular, if all antichains of $B(X)$ are countable, then all subspaces of $X$ have countable $\pi$-weight and hence $X$ is hereditarily separable. A familiar example can be used to show that the above result cannot be improved to an equality: the Alexandroff-Urysohn "double-arrow" space (which is the same as the top and bottom of the lexicographically ordered unit square), is a compact zero-dimensional space which has an antichain of $c$ clopen sets. Furthermore, this space is hereditarily separable and first countable, and hence its hereditary $\pi$ weight is $\omega$. This example is also discussed in $9 \mathrm{C}$ of $[\mathbf{E}]$.

We will now establish a relation between chains of $B(X)$ and cardinal invariants of the space $X$.

2.1 THEOREM. Let $\kappa$ be an infinite cardinal. If $B(X)$ contains a chain of cardinality $\kappa$ then $X$ contains a totally separated subspace of cardinality $\kappa$, and $X \times X$ contains $a$ discrete subset of cardinality $\kappa$. In particular, $h(B(X))<s(X \times X)$.

Proof. Let $\Lambda$ be a chain in $B(X)$ with $|\Lambda|=\kappa$. By deleting $\varnothing$ if necessary we may assume that every member of $\Lambda$ is nonempty. Let $A \in \Lambda$. We claim that $\cup\{B: B \in \Lambda$ and $B \subsetneq A\} \subsetneq A$. For $A$ is compact, being closed in $X$, and so, if the preceding union where equal to $A$ it follows that there would exist a finite number $B_{1}, B_{2}, \cdots, B_{n}$ of members of $\Lambda$ with $B_{i} \subsetneq A$ for all $i=1,2, \ldots, n$ such that $B_{1} \cup B_{2} \cup \cdots \cup B_{n}=A$. But $\Lambda$ is a chain under inclusion so there is a largest member among $B_{1}, B_{2}, \ldots, B_{n}$, say $B_{j}$. Thus $B_{j}=A$. But this is impossible, since $B_{j}$ is a proper subset of $A$. This proves our claim. Thus for each $A$ in $\Lambda$ we may

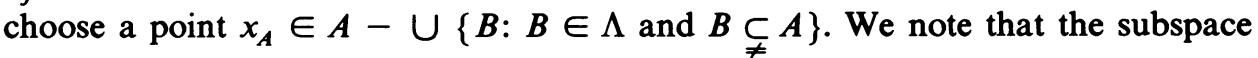


$S=\left\{x_{A}: A \in \Lambda\right\}$ is totally separated by $\Lambda$ under inclusion-in fact, for all $A$ in $\Lambda$ we have $\left\{x_{B}: B \in \Lambda\right.$ and $\left.B \subseteq A\right\}=A \cap S$, which implies that the set $\left\{x_{B}: B \in \Lambda\right.$ and $B \subseteq A\}$ is open in $S$. Thus $S$ is a totally separated subspace of $X$ having cardinality $\kappa$. This establishes the first part of the theorem.

Now, we can repeat the above argument applied to the chain $\Lambda^{*}=\{X-A$ : $A \in \Lambda$ \} (after deleting $X$ from $\Lambda$ if necessary to ensure that each member of $\Lambda$ is proper). The result is a set of points $\left\{y_{A}: A \in \Lambda\right\}$ such that, for all $A, y_{A} \in X-A$, and $y_{A} \notin X-B$ for any $B$ in $\Lambda$ with $X-B \subsetneq X-A$. That is, $y_{A} \notin A$ and $y_{A} \in B$ for all $B$ in $\Lambda$ such that $A \subsetneq B$. We now claim that the set $D=\left\{\left(x_{A}, y_{A}\right)\right.$ : $A \in \Lambda\}$ is a discrete subset of $X \times^{\mp} X$, proving that $X \times X$ has a discrete subset of cardinality $\kappa$ as desired. This follows from the fact that $A \times(X-A)$ is open in $X \times X$ and $A \times(X-A) \cap D=\left\{\left(x_{A}, y_{A}\right)\right\}:$ for, if $B \subset A$ then $y_{B} \in A$ and so $\left(x_{B}, y_{B}\right) \notin A \times(X-A)$, while if $A \subsetneq B$ then $x_{B} \notin A$ and so again $\left(x_{B}, y_{B}\right) \notin A$ $\times(X-A)$. This completes the proof.

An easy example shows that the inequality in our theorem cannot be improved to an equality: Let $X$ be the one-point compactification of the discrete space of cardinality $\kappa$. Since all clopen subsets of $X$ are either finite or cofinite, it follows that all chains in $B(X)$ are countable. However $X \times X$, and indeed $X$ itself, contains a discrete subset of cardinality $\kappa$.

It is worth noting that the existence of a discrete subset of $X$ of cardinality $\kappa$ does not follow from the existence of a chain in $B(X)$ of cardinality $\kappa$. The double-arrow space serves as an example here also; it contains a chain of $c$ clopen sets but contains no uncountable discrete subsets, since it is hereditarily separable.

As for discrete subsets of $X$ we have the following simple result.

2.2 TheOREM. Let $X$ be a compact zero-dimensional space and let $\kappa$ be an infinite cardinal. Then $X$ contains $a$ discrete subset of cardinality $\kappa$ if and only if $B(X)$ contains a strong antichain of cardinality $\kappa$.

Proof. Suppose $X$ contains a discrete subset $D$ with $|D|=\kappa$. Then for each $x$ in $D$ there is a clopen set $A_{x}$ in $X$ such that $A_{x} \cap D=\{x\}$. Clearly the collection $\Lambda=\left\{A_{x}: x \in D\right\}$ is a strong antichain in $B(X)$, since, for any $x$ in $D$ and any finite subset $F$ of $D-\{x\}$, we have $x \in A_{x}-\cup\left\{A_{y}: y \in F\right\}$ and so $A_{x} \nsubseteq$ $\cup\left\{A_{y}: y \in F\right\}$. Thus $\Lambda$ is a strong antichain of cardinality $\kappa$.

Conversely, suppose $\Lambda$ is a strong antichain in $B(X)$ of cardinality $\kappa$. Let $A$ be any member of $\Lambda$. The family of clopen sets $\{A-B: B \in \Lambda-\{A\}\}$ has the finite intersection property, since $A$ is not contained in the union of any finite number of members of $\Lambda-\{A\}$. Therefore, by compactness, $\cap\{A-B: B \in \Lambda-\{A\}\} \neq$ $\varnothing$. Choose a point $x_{A}$ in that intersection. Doing this for each $A$ in $\Lambda$ we construct a subset $D=\left\{x_{A}: A \in \Lambda\right\}$ of $X$ such that $A \cap D=\left\{x_{A}\right\}$ for all $A$ in $\Lambda$. Thus the set $D$ is a discrete set of cardinality $\kappa$.

Combining 2.1 and 2.2 and using the obvious fact that $X \times X$ contains a discrete set of cardinality $\kappa$ whenever $X$ does, we have the following corollary.

2.3 Corollary. If $X \times X$ contains no discrete subsets of cardinality $>\kappa$ then all chains and all strong antichains of $B(X)$ have cardinality at most $\kappa$. 
REMARK. The conclusion of 2.2 has an obvious generalization to arbitrary compact spaces. One replaces the concept of a strong antichain of clopen sets by a set of pairs $\left\{\left(F_{i}, G_{i}\right): i \in I\right\}$ where $F_{i}$ is closed and $G_{i}$ is open and $F_{i} \subseteq G_{i}$ and such that for any $i$ and any finite subset $J$ of $I-\{i\}, F_{i} \& \cup\left\{G_{j}: j \in J\right\}$. There does not seem to be any natural generalization of 2.1 to arbitrary compact spaces.

\section{REFERENCES}

[BK] J. Baumgartner and P. Komjath, Boolean algebras in which every chain and every antichain is countable (to appear).

[E] R. Engleking, Outline of general topology, North-Holland, Amsterdam, 1968.

[IN] M. Ismail and P. Nyikos, Countable small rank and cardinal invariants II (preprint).

[J] I. Juhasz, Cardinal functions in topology, Math. Centre Tracts, No. 34, Math. Centre, Amsterdam, 1971.

Department of Mathematics, University of Manitoba, Winnipeg, Manitoba, Canada R3T 2 N2

Department of Mathematics, University of Winnipeg, Winnipeg, Manitoba, Canada R3B 2E9 